\title{
Simulation of Sound Emitted from Collision of Droplet with Shallow Water by the Lattice Boltzmann Method
}

\author{
Shinsuke Tajiri ${ }^{1}$, Michihisa Tsutahara ${ }^{1}$, and Hisao Tanaka ${ }^{2}$ \\ ${ }^{1}$ Graduate school of Engineering, Kobe University \\ Rokko, Nada-ku, Kobe 657-8501 Japan \\ tajiri@mh-1.scitec.kobe-u.ac.jp, \\ tutahara@mech.kobe-u.ac.jp \\ ${ }^{2}$ Universal Shipbuilding Corporation \\ 1-3 Kumozu-kokan-cho, Tsu, Mie 514-0398, Japan \\ tanaka-hisao@u-zosen.co.jp
}

\begin{abstract}
The sound emitted from splash of water droplet colliding with shallow water is simulated by the finite difference lattice Boltzmann method. Twoparticle immiscible fluid model is used, and the under water sound is considered by introducing the elasticity for the liquid phase. After the collision, sounds propagating into the gas and liquid phases are successively detected. The directivity of the sound is shown to depend on the depth of the water.
\end{abstract}

Keywords: Finite difference lattice Boltzmann method, Aero-acoustics, Twophase flow, Two-fluid interface.

\section{Introduction}

The sounds generated at interfaces between liquid and gas are commonly heard in ordinary life such as sound from raindrops and so on. But the mechanism is still not clear because the scales of motion of the interface and sound are very different and the experimental investigations are still hard. It is also still very difficult to calculate sound waves and deformation of interfaces simultaneously. As to the fluid dynamic sound, direct simulations have become popular by using high-resolution schemes.

On the other hand, many calculation methods for interfaces have been developed. MAC (Marker And Cell) [1], VOF (Volume Of Fluid) [2] and LS (Level-Set) [3] methods are widely used. However, these methods sometimes suffer the lack of conservation of volume. CIP (Cubic- Interpolated Pseudo-particle [4]) has been considered to reduce the diffusivity at the interface.

Immiscible models based on the LBM (Lattice Boltzmann Method), which are excellent in conservation of volume or mass, have been developed $[5,6]$ for simulation of multi-phase flows. In this paper, we present a two-particle model for two-phase flows, gas and liquid, considering large density difference, surface tension effect, and compressibility of the liquid phase. 


\section{Basic Equations}

\subsection{Discrete BGK Equation}

The basic equation for the finite difference lattice Boltzmann method is the following discrete BGK equation [7]

$$
\frac{\partial f_{i}^{k}}{\partial t}+c_{i \alpha} \frac{\partial f_{i}^{k}}{\partial x_{\alpha}}=-\frac{1}{\tau}\left(f_{i}^{k}-f_{i}^{e q k}\right)
$$

where $f_{i}$ is the distribution function which is the number density of the particle having velocity $\mathrm{c}_{i \alpha}$ and subscripts $i$ represents the direction of particle translation and $\alpha$ is the Cartesian co-ordinates. $f_{i}^{e q}$ is the local equilibrium distribution function. The term on the right hand side represents the collision of particles and $\tau$ is called the single relaxation time factor. Superscript $k$ represents gas and liquid phases, and we will use two particle model and $k=G$ represents gas phase and $k=L$ liquid phase, respectively.

Macroscopic variables, density $\rho$, flow velocity $\mathbf{u}$, and internal energy $e$ are obtained by

$$
\begin{gathered}
\rho^{k}=\sum_{i}^{m_{p}} f_{i}^{k}=\sum_{i}^{m_{p}} f_{i}^{e q k} \\
\rho \mathbf{u}=\sum_{i, k}^{m_{p}} f_{i}^{k} \mathbf{c}_{i}=\sum_{i, k}^{m_{p}} f_{i}^{e q k} \mathbf{c}_{i} \\
\frac{1}{2} \rho u^{2}+\rho e=\sum_{i, k}^{m_{p}} \frac{1}{2} f_{i}^{k} c_{i}^{2}=\sum_{i, k}^{m_{p}} \frac{1}{2} f_{i}^{e q k} c_{i}^{2}
\end{gathered}
$$

where $m_{p}$ represents the number of the particles. The pressure $P=\rho e$ and sound speed in gas phase $c_{s G}=\sqrt{2 e}$ for two-dimensional flows.

\subsection{Interface Treatment}

In order to obtain sharp interface for immiscible two-particle model, artificial separation techniques [5] are sometimes introduced. In this study, phase separation or recolor technique by Latva-Kokko et al [8,9] is employed.

In this technique, an additional term is introduced to the discrete BGK equation as

$$
\frac{\partial f_{i}^{k}}{\partial t}+c_{i \alpha} \frac{\partial f_{i}^{k}}{\partial x_{\alpha}}=-\frac{1}{\tau}\left(f_{i}^{k}-f_{i}^{e q k}\right)+\left(f_{i}^{k}-f_{i}^{\prime k}\right)
$$

where $f_{i}^{\prime k}$ is re-distributed function calculated from the gradient of the interface. $f_{i}^{\prime k}$ is given by 


$$
\begin{aligned}
& f_{i}^{\prime G}=\frac{\rho_{G}}{\rho_{G}+\rho_{L}}\left(f_{i}^{G}+f_{i}^{L}\right)+\left.\kappa \frac{\rho_{G} \rho_{L}}{\left(\rho_{G}+\rho_{L}\right)^{2}}\left(f_{i}^{e q G(0)}+f_{i}^{e q L(0)}\right) \cos \varphi\right|_{i} \\
& f_{i}^{\prime L}=\frac{\rho_{L}}{\rho_{G}+\rho_{L}}\left(f_{i}^{G}+f_{i}^{L}\right)-\left.\kappa \frac{\rho_{G} \rho_{L}}{\left(\rho_{G}+\rho_{L}\right)^{2}}\left(f_{i}^{e q G(0)}+f_{i}^{e q L(0)}\right) \cos \varphi\right|_{i}
\end{aligned}
$$

where $\kappa$ is a parameter to control the thickness of the diffusive interface and $f_{i}^{e q k(0)}$ is the equilibrium distribution function for velocity zero considering the natural or pure diffusion. Each sum of $f_{i}^{\prime G}, f_{i}^{\prime L}$ is not changed at the collision, and then the density, the momentum and the energy are conserved. $\varphi$ is the angle between the gradient of the density and the particle velocity and given by

$$
\begin{gathered}
\left.\cos \varphi\right|_{i}=\frac{\mathbf{G} \cdot \mathbf{c}_{i}}{|\mathbf{G}| \cdot\left|\mathbf{c}_{i}\right|} \\
\mathbf{G}(\mathbf{x})=\sum_{i} \mathbf{c}_{i}\left[\rho^{G}\left(\mathbf{x}+\mathbf{c}_{i}\right)-\rho^{L}\left(\mathbf{x}+\mathbf{c}_{i}\right)\right]
\end{gathered}
$$

\subsection{Introduction of External Forces}

External forces are introduced [10] to the discrete BGK equation as

$$
\frac{\partial f_{i}^{k}}{\partial t}+c_{i \alpha} \frac{\partial f_{i}^{k}}{\partial x_{\alpha}}=-\frac{1}{\tau}\left(f_{i}^{k}-f_{i}^{e q k}\right)+\frac{\left(c_{i \alpha}-u_{\alpha}\right) F_{\alpha}^{k}}{R T} f_{i}^{e q k}
$$

where $R$ is the gas constant and $T$ is the absolute temperature. Alternatively, the force is introduced to the equilibrium distribution function as an impulsive force, and we employ the latter technique

The equilibrium distribution function $f_{i}^{e q k}=f_{i}^{e q k}\left(t, \rho^{k}, u_{\alpha}\right)$ is modified by the external force $F_{\alpha}$ as

$$
\begin{gathered}
\rho \rightarrow \rho \\
\rho u_{\alpha} \rightarrow \rho\left(u_{\alpha}+\tau F_{\alpha}\right) \\
\frac{1}{2} \rho u^{2}+\rho e \rightarrow \frac{1}{2} \rho u^{2}+\rho(e-W)
\end{gathered}
$$

where $W$ is the work done by the external force, and give by

$$
W=\frac{1}{2} \tau^{2} F^{2}
$$

\subsection{Surface Tension}

We employ a model proposed by Gunstensen [11] and Continuum Surface Force (CSF) method [12]. In CSF, the surface tension force $\mathbf{F}_{S}$ is given by

$$
\mathbf{F}_{S}=\sigma \mathbf{K} \hat{\mathbf{n}}
$$


where $\sigma$ is the surface (interfacial) tension coefficient $\mathbf{K}$ is the curvature of the interface, $\hat{\mathbf{n}}$ is the normal unit vector of the interface, and $\hat{\mathbf{n}}(\mathbf{x})=\mathbf{n}(\mathbf{x}) /|\mathbf{n}(\mathbf{x})|$. The normal vector on the interface $\mathbf{n}(\mathbf{x})$ is calculated by

$$
\mathbf{n}(\mathbf{x})=\frac{\partial\left(\rho^{G}(\mathbf{x})-\rho^{L}(\mathbf{x})\right)}{\partial \mathbf{x}}
$$

The curvature $\mathbf{K}$ is also calculated by

$$
\mathbf{K}=-(\nabla \cdot \hat{\mathbf{n}})=\frac{1}{|\mathbf{n}|}\left[\left(\frac{\mathbf{n}}{|\mathbf{n}|} \cdot \nabla\right)|\mathbf{n}|-(\nabla \cdot \mathbf{n})\right]
$$

\subsection{Model for Two Fluids with Large Density Difference}

He et al [13] have proposed a large density difference fluid model up to about 40, and Inamuro et al [14] have proposed a model with density difference up to 1000 . But the fluid of liquid phase is completely incompressible in Inamuro's model, and the sound in liquid phase can not simulated by his model. Therefore we will propose a novel model for two-phase flows with large density difference. The density difference is realized by changing the acceleration, and this effect is also introduced to the model by impulsive force for each particle

$$
\begin{aligned}
\mathbf{F}_{i n} & =-\mathbf{a}+\mathbf{a}^{\prime} \\
& =-\left(-\frac{1}{\rho} \frac{\partial P}{\partial \mathbf{x}}+\frac{\mu}{\rho} \nabla^{2} \mathbf{u}\right)+\left(-\frac{1}{m \rho} \frac{\partial P^{\prime}}{\partial \mathbf{x}}+\frac{\mu^{\prime}}{m \rho} \nabla^{2} \mathbf{u}\right)
\end{aligned}
$$

where $P^{\prime}$ is the effective pressure, and $\mathbf{a}$ is the acceleration to cancel the original force term, $\mathbf{a}^{\prime}$ is newly introduced acceleration due to the density and the viscosity of considering fluid. $m$ represents an averaged density and $\mu^{\prime}$ is an averaged viscosity

$$
m=\frac{\sum_{k=G, L} m^{k} \rho^{k}}{\sum_{k=G, L} \rho^{k}}, \quad \mu^{\prime}=\frac{\sum_{k=G, L} \mu^{k} \rho^{k}}{\sum_{k=G, L} \rho^{k}}
$$

The density ratio of the two fluids is given as $m_{L} / m_{G}$, and in case of water and air, it is about 800 , and the ratio of viscosity is about 70 and they change continuously across the interface.

\subsection{Compressibility of Liquid}

A simple model of bulk elasticity is introduced to consider the compressibility of the liquid as

$$
P^{\prime}=P_{0}+\beta \frac{\rho-\rho_{0}}{\rho_{0}}
$$


where $\beta$ is a parameter to control the elasticity and corresponds the bulk (elasticity) modulus and $P_{0}$ is a reference pressure and $\rho_{0}$ is a reference density and is fixed to unity in this study. In liquid phase, we do not consider the temperature change.

\section{Two-Dimensional Thermal Model}

\subsection{Two-Dimensional 21 Velocity Model}

In this study, two-dimensional thermal 21-velocity (D2Q21) model $[15,16]$ is used, whose equilibrium distribution function is given by

$$
\begin{aligned}
f_{i}^{(0)}= & F_{i} \rho\left[1-2 B\left(\mathbf{c}_{i} \cdot \mathbf{u}\right)+2 B^{2}\left(\mathbf{c}_{i} \cdot \mathbf{u}\right)^{2}+B(\mathbf{u} \cdot \mathbf{u})\right. \\
& \left.-4 / 3 B^{3}\left(\mathbf{c}_{i} \cdot \mathbf{u}\right)^{3}-2 B^{2}\left(\mathbf{c}_{i} \cdot \mathbf{u}\right)(\mathbf{u} \cdot \mathbf{u})\right]
\end{aligned}
$$

The velocity set and the coefficients are shown in Fig.1, Tables 1 and 2, respectively.

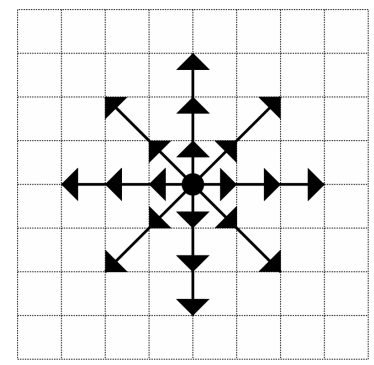

Fig. 1. Distribution of particles of D2Q21 model

Table 1. Velocity set in D2Q21 model

\begin{tabular}{|c|c|c|}
\hline$i$ & Velocity vector & $\mid \mathbf{c}$ \\
\hline 1 & $(0,0)$ & 0 \\
\hline $2-5$ & $(1,0),(0,1),(-1,0),(0,-1)$ & 1 \\
\hline $6-9$ & $(2,0),(0,2),(-2,0),(0,-2)$ & 2 \\
\hline $10-13$ & $(3,0),(0,3),(-3,0),(0,-3)$ & 3 \\
\hline $14-17$ & $(1,1),(-1,1),(-1,-1),(1,-1)$ & $\sqrt{2}$ \\
\hline $18-21$ & $(2,2),(-2,2),(-2,-2),(2,-2)$ & $2 \sqrt{2}$ \\
\hline
\end{tabular}


Table 2. The coefficients $F_{\mathrm{i}}$ and B in D2Q21 model

\begin{tabular}{|c|c|}
\hline$i$ & $\mathrm{~F}_{\mathrm{i}}$ \\
\hline 1 & $1+\frac{5}{4 B c^{2}}\left(\frac{17}{96 B^{2} c^{4}}+\frac{35}{48 B c^{2}}+\frac{49}{45}\right)$ \\
\hline $2-5$ & $-\frac{1}{8 B c^{2}}\left(\frac{13}{16 B^{2} c^{4}}+\frac{71}{24 B c^{2}}+3\right)$ \\
\hline $6-9$ & $\frac{1}{16 B c^{2}}\left(\frac{5}{16 B^{2} c^{4}}+\frac{25}{24 B c^{2}}+\frac{3}{5}\right)$ \\
\hline $10-13$ & $-\frac{1}{24 B c^{2}}\left(\frac{1}{16 B^{2} c^{4}}+\frac{1}{8 B c^{2}}+\frac{1}{15}\right)$ \\
\hline $14-17$ & $\frac{1}{4 B^{3} c^{6}}\left(\frac{B c^{2}}{3}+\frac{1}{8}\right)$ \\
\hline $18-21$ & $-\frac{1}{1536 B^{3} c^{6}}\left(2 B c^{2}+3\right)$ \\
\hline
\end{tabular}

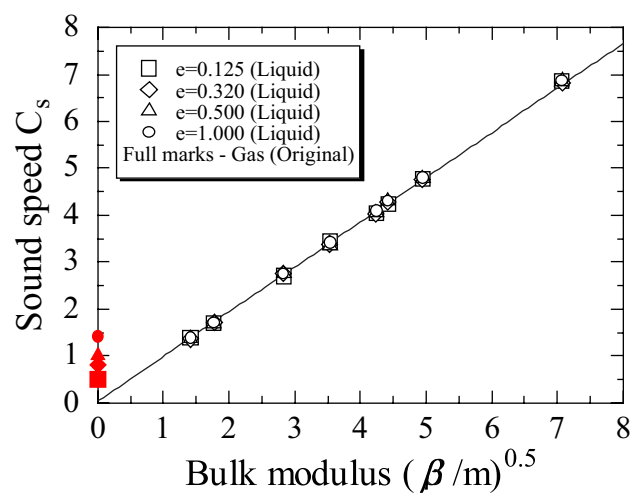

Fig. 2. Sound speed versus bulk modulus by using D2Q21 model on internal energies $e_{0}=0.125$, $0.32,0.5$, and 1. Full marks represent the sound speed of original FDLBM on each internal energy. The sound speed of liquid depends on only bulk elasticity modulus.

\subsection{Sound Speed of Liquid Phase}

The sound speed of the liquid phase is given as

$$
c_{s L}=\sqrt{\frac{\Delta P^{\prime}}{\Delta \rho}}=\sqrt{\frac{\beta}{m}}
$$

In order to check the sound speed, we calculated very weak pressure wave by a piston problem. The results are shown in Fig. 2, and the relation given above is shown to 
be satisfied. This result does not depend on numerical scheme. If we consider a system consists of water and air, the ratio of both sound speeds is about 4.4 and $\sqrt{\beta / m}$ can be adequately chosen.

\section{Sound Generated by Collision of a Water Drop on a Shallow Water}

The sound generated by rain drops is a very well-known sound, and intensive studies have been done [17]. In this repot, we calculated the sound generated by collision of a water drop to shallow water using the above mentioned model. The reason why we chose this problem is that the results, such as splash shape except sound, can be compared with other simulation results.

In this calculation, we did not impose the gravitational force, because some internal compression waves are generated just after the gravitational force is imposed. Instead, the initial velocity was imposed as shown in Fig. 3. Space differential is discretized by the third order upwind scheme (UTOPIA) and time integration is done by the second order Runge-Kutta method. The parameters of the calculation are given as follows.

The number of grids is $503 \times 401$ and the minimum grid size is $2 \times 10^{-5}$. Time increment is set $2 \times 10^{-6}$. The density ratio $m_{L} / m_{G}=1000$, kinematic viscosity of gas $v_{G}=1 \times 10^{-6}$, and the bulk elastic modulus of liquid is 6400 . The phase separation coefficient $\kappa=0.9$, the surface tension coefficient $\sigma=1 \times 10^{-7}$, the droplet diameter $D=2 \times 10^{-3}$, the water depth $D_{f}=0.075 D$, and the initial velocity of the droplet $U=0.02$. It should be noted here that these parameters are all non-dimensional values based on the minimum particle speed $|\mathbf{c}|=1$, the reference time $t=1$, and the reference length $|\mathbf{c}| / t=1$.

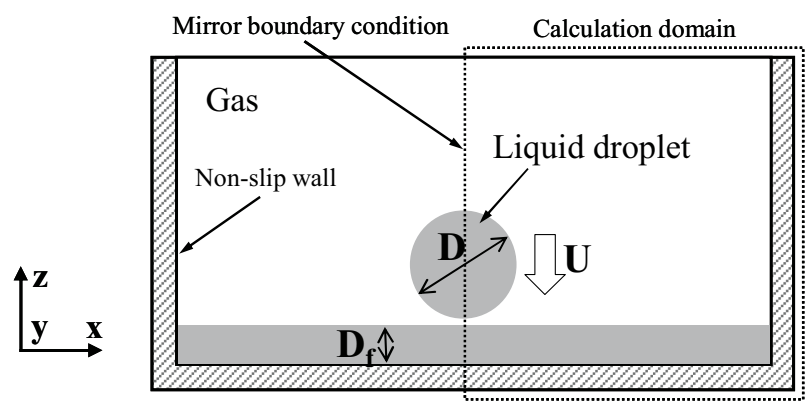

Fig. 3. Calculation domain for impact of a drop on a thin film of the same liquid. Mirror boundary condition was employed for the central domain, and the upper boundary condition was free boundary. 


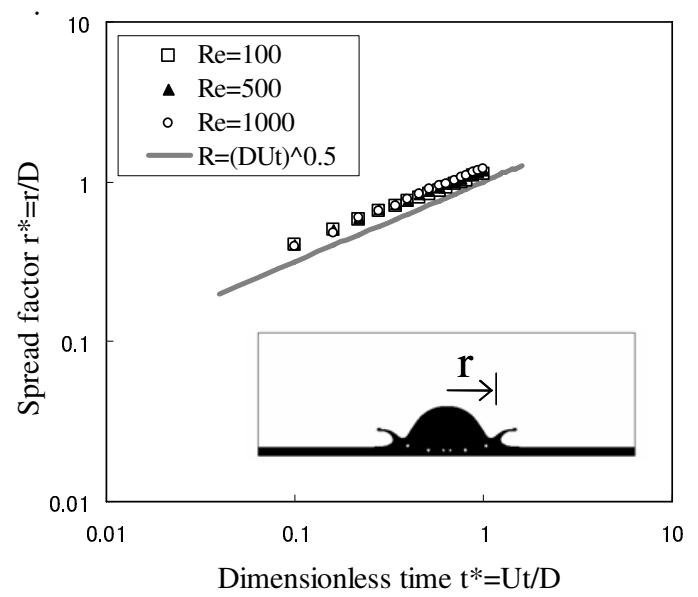

Fig. 4. Log-log plot of the spread factor $r^{*}$. The solid line corresponds to the power law $R=\sqrt{D U t}$.
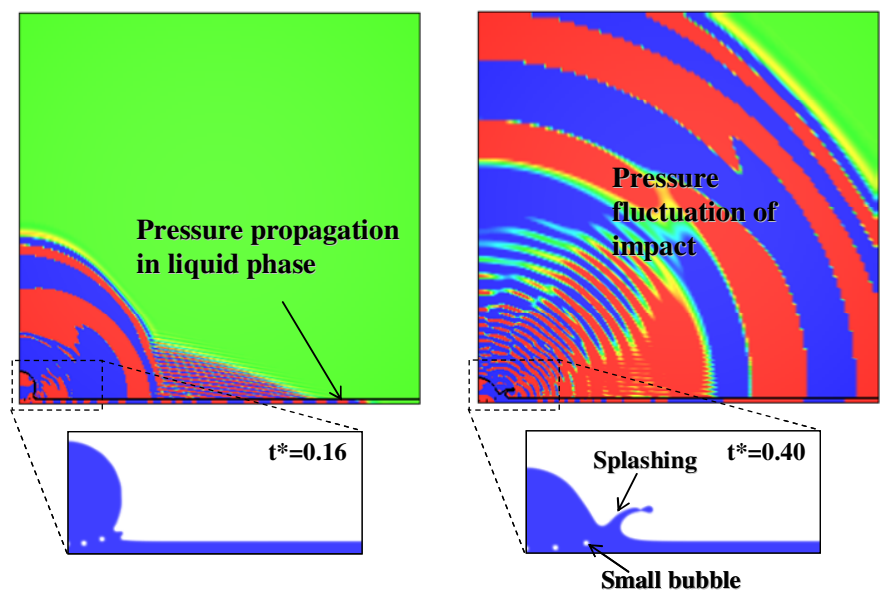

Fig. 5. Density field and pressure distribution for Reynolds number $\mathrm{Re}=1000$

Figure 4 shows the relationship between another non-dimensional time $t^{*}=U t / D$ and space factor $r^{*}=r / D$, where $U$ is the initial velocity of the drop, $D$ is the diameter of the drop and $r$ is the radius of the splash root. The relationship fits well to a power law.

The sound generated at the collision is shown in Fig. 5. Sounds propagating into the gas phase and also into the liquid phase are simultaneously shown there. The sound generated by the deformation of the drop (round shaped sound) and generated by the underwater sound (sound speed is 4.4 times larger than the former) are clearly 


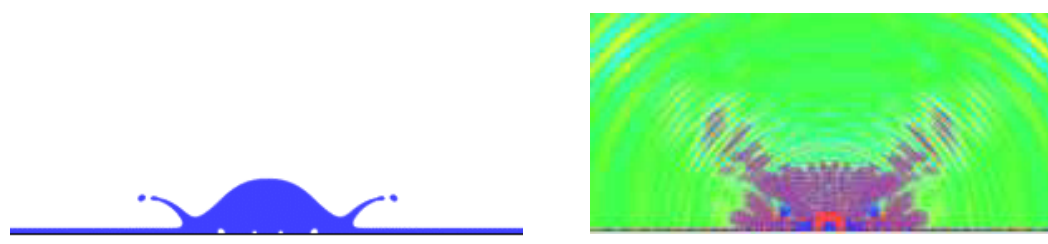

(a) $D_{f}=0.075 D$

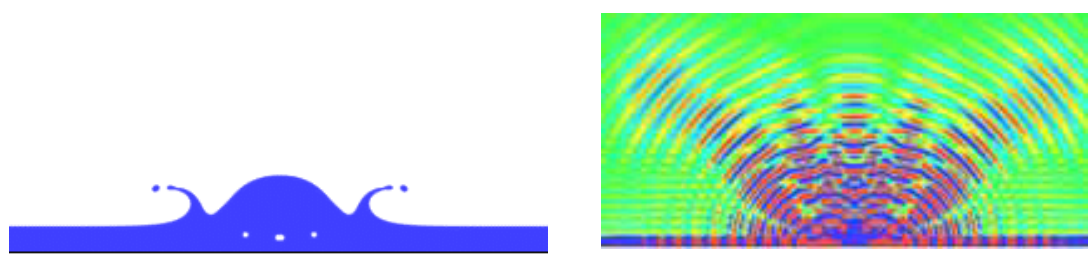

(b) $D_{f}=0.3 D$

Fig. 6. The directivity of sound propagating into gas phase for different water depth

presented. It should be noted that small gas bubbles are also generated and caught by water. Figure 6 shows that the sound propagating into gas phase has complicated directivity, and that the directivity depends on the depth of the water.

\section{Conclusion}

A two-phase-flow model with large density difference and including the elasticity of liquid is proposed and simulation of sound generated when a water droplet collides with shallow water was performed. The sounds propagating into gas and liquid phases were successively simulated.

\section{References}

1. Harlow, F.H., Welch, J.E.: Numerical calculation of time-dependent viscous incompressible flow of fluid with free surface. Physics of Fluids 8, 2182-2189 (1965)

2. Hirt, C.W., Nichols, B.D.: Volume of fluid (VOF) method for the dynamics of free boundaries. Journal of Computational physics 39, 201-225 (1981)

3. Sussman, M., Smereka, P.: Axisymmetric free boundary problem. Journal of Fluid Mechanics 341, 269-294 (1997)

4. Yabe, T., et al.: The constrained Interpolation profile method for multiphase analysis. Journal of Computational physics 169(2), 556-593 (2001)

5. Shan, X., Chen, H.: Simulation of non-ideal gases and liquid-gas phase transition by the lattice Boltzmann equation. Physical Review E 49, 2941-2948 (1994)

6. Swift, M.R., Osborn, W.R., Yeomans, J.M.: Lattice Boltzmann simulation of non-ideal fluids. Physical Review Letter 75, 830-833 (1995) 
7. Succi, S.: The lattice Boltzmann equation for fluid dynamics and beyond, Oxford, pp. 70$73(2001)$

8. Latva-Kokko, M., Rothman, D.H.: Diffusion properties of gradient-based lattice Boltzmann models of immiscible fluids. Physical Review E 71, 056702 (2005)

9. Latva-Kokko, M., Rothman, D.H.: Static contact angle in lattice Boltzmann models of immiscible fluids. Physical Review E 72, 046701 (2005)

10. He, X., Shan, X., Doolen, G.D.: Discrete Boltzmann equation model for nonideal gases. Physical Review E 57, 13-16 (1998)

11. Gunstensen, A.K., Rothman, D.H., Zaleski, S., Zanetti, G.: Lattice Boltzmann model of immiscible fluid. Physical Review A 43, 4320-4327 (1991)

12. Brackbill, J.U., Kothe, D.B., Zemach, C.: A continuum method for modeling surface tension. Journal of Computational Physics 100, 335-354 (1992)

13. He, X., Chen, S., Zhang, R.: A lattice Boltzmann Scheme for Incompressible Multiphase Flow and Its Application in Simulation of Rayleigh-Taylor Instability. Journal of Computational Physics 152, 642-663 (1999)

14. Inamuro, T., Ogata, T., Tajima, S., Konishi, N.: A lattice Boltzmann method for incompressible two-phase flows with large density differences. J. Computational Physics 198, 628-644 (2004)

15. Tsutahara, M., Kurita, M., Kataoka, T.: Direct simulation of Aeolian tone by the finite difference Lattice Boltzmann method. Computational Fluid Dynamics 2002, 508-513 (2003)

16. Tsutahara, M., Kataoka, T., Shikata, K., Takada, N.: New model and scheme for compressible fluids of the finite difference lattice Boltzmann method and direct simulations of aerodynamic sound. 37, 79-89 (2007)

17. Pumphrey, H.C., Crum, L.A., Bjomo, L.: Underwater sound produced by indivisual drop impacts and rainfall. Journal of Acoustical Society of America 85(4), 1518-1526 (1989) 